\title{
EVALUATION OF WHEAT SEED SEPARATION PERFORMANCES FOR NEW DESIGN OF ROTATING CYLINDRICAL SIEVE, EQUIPPED WITH CUSTOMIZABLE HOMOGENIZATION COIL
}

Florin Nenciu ${ }^{1}$, Costin Mircea ${ }^{1}$, Valentin Vladut ${ }^{1}$, Nastasia Belc ${ }^{2}$, Mariana Berca Lavinia ${ }^{2}$

${ }^{1}$ National Institute of Research-Development for Machines and Installations Designed to Agriculture and Food Industry, Romania; ${ }^{2}$ National Research and Development Institute for Food Bioresources , Romania

florin_nenciu2000@yahoo.com , costinmircea@yahoo.com

\begin{abstract}
Organic farming practices impose restrictions on the use of chemicals in the technological phases of cultivation and processing of cereals. The restrictions may bring several challenges regarding the appropriate separation of wheat from contaminants and the efficiency of sorting the seeds by quality classes. Seed cleaning and sorting equipment working with rotating sieves show several advantages, consisting in a significant reduction of noise, increased durability of the components and the possibility to exclude other more complex devices from the technological flow. The main objective of the study was to improve the processes efficiency of the rotary grain cylindrical separators that are using small sieves, by installing a novel customizable subassembly. The equipment is designed in the form of an inner coil and has the role of breaking the immobile core of seed found on the sieve, to increase the aggressiveness of seed mixing and to better spread the seeds on the sieve surface. Comparatively evaluating the equipment during operation with and without the newly created subassembly, we were able to assess the distribution of the weights along the length of the cylindrical sieve and determine the optimal processing parameters. The working regime that allowed obtaining both the maximum amount of processed material and also a superior quality of the seeds, showed to be strongly dependent on the inclination angle of the sieve, the sieve speed, and the impurities content from the raw material.
\end{abstract}

Keywords: cylindrical sieve, wheat seed conditioning.

\section{Introduction}

Wheat seed sorting equipment, using cylindrical rotary sieves, has several advantages in comparison to other seed separation devices. Cylindrical sorting equipment can be used with high efficiencies in situations where a more aggressive processing of the seeds is needed, considering that a more intense mixing of particles can be achieved. It has a relatively simple construction, small size and low power consumption, has less maintenance and repairing costs compared to flat vibrating sieves and produce lower noise levels [1;2]. Cylindrical sieves do not need to be balanced, as in the case of flat sieves, their subassemblies are more compact, have a uniform movement and simple transmission [3].

However, cylindrical sieves can only operate in slow working regimes, which can successfully ensure the sliding movement of wheat seeds inside the sieve, and this reduces the productivity levels of any equipment that uses this technology [4]. The increase of the sieve speed in order to enhance the productivity, leads to the transition to another type of movement due to the inertia, where the seeds tend to stick to the sieve walls, and leading to a relative resting phase that makes separation difficult $[4 ; 5]$.

The separation process is a complex phenomenon, being influenced by many factors, such as the specific feeding rate, the small seed content (considered impurities) from the row material (\%); the physical characteristics of the granular material, the characteristics of the holes of the sieve, the kinematic operating regime of the sieve and the angle of inclination of the sieve [5;6].

The problem addressed is the low efficiency of equipment that uses short length sieves, for the separation of wheat seeds. In order to improve the separation performance, it is necessary to adjust submodules that increase the agitation of the seeds, break the immobile nucleus created on the sieve, and increase the separation in time.

In order to analyze the behavior of wheat seeds on the cylindrical surface of the sieve and to come up with improvements in operation, an experimental stand has been designed and built to test equipment parameters functioning, with some novel subassemblies that can increase the yield.

In order to eliminate the main disadvantages of cylindrical sieves - speed and low productivity - an innovative model of inner coil was designed, to improve the movement of wheat seeds during the equipment operation. A pilot experimental stand was designed, capable to determine the separation distribution along the length of the sieve, in both horizontal and angular plane depending on some adjustable parameters. 
These parameters were varied to identify the separation characteristics, and for the first stage of assessments the following criterion have been chosen: the angle of inclination of the sieve to the horizontal, the speed of the sieve, the pitch of the inner coil, and the distance between the components of the coil.

\section{Materials and methods}

The experimental pilot stand, depicted in Figure 1, is placed on a support for precise adjustment of the equipment height (1), which holds the stand housing (2). In the lower part of the stand there is located a sub-assembly, designed for guiding the seeds in the collecting boxes (3). The feeding hopper (4) adjusts the material flow that enters the experimental stand, operating on a bunker shutter adjustment system (5). The separation subassembly consists of a sieve, which has a customizable and adaptable inside coil (6). The sieve and the coil are being driven in opposite directions by the gear system (7), coordinated by the electric motor (13). The seeds that pass through the sieve holes are oriented towards eighteen collection boxes located in the vertical plane (8) and other eighteen collection boxes that take over the seeds in the horizontal plane (9).

It can be seen that the horizontal drawer (9) has the role of collecting all the small impurities passing through the sieve holes, sorted along the sieve, while the vertical drawer (8) has the role of collecting all impurities which are sorted radially, being divided into intervals, between -60 and 150 degrees. The large number of collection boxes arranged both radially and horizontally (thirty-six in total), generates accurate results on the distribution of impurities along the cylindrical sieve. The large seeds (wheat without impurities) are collected at the end of the sieve, in two collection boxes, intended for seeds that do not fall through the separation holes.

The inclination angles of the installation are adjusted using the adjustment systems (10 and 12), while the seeds locked on the sieve holes are pushed back by the brush (11).
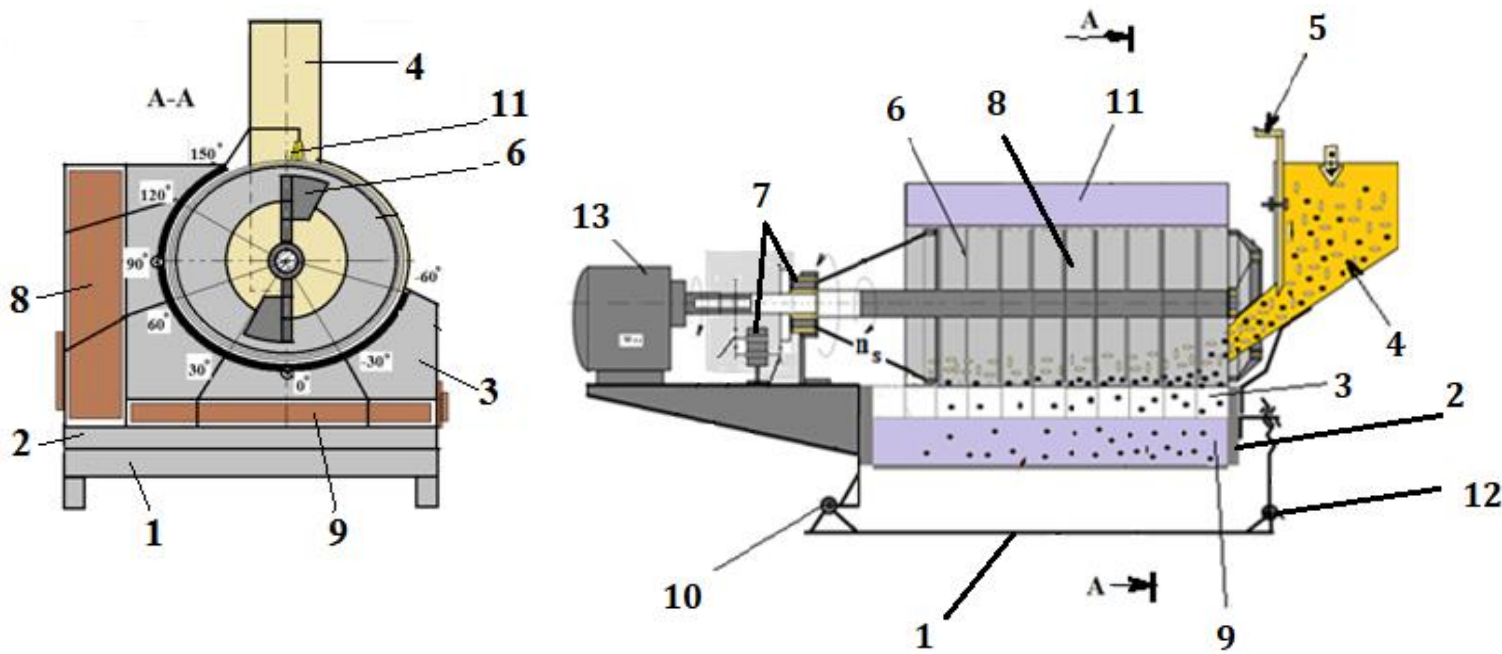

Fig. 1. Experimental pilot stand: 1 - support for precise adjustment of the height; 2 - stand housing; 3 - collection boxes; 4 - feeding hopper; 5 - bunker shutter adjustment system; 6 - system consisting of a rotating sieve and a coil; 7 - transmission gear system; 8 - vertical collection boxes;

9 - horizontal collection boxes; 10 - sieve angle adjustment system; 11 - cleaning brush of the remaining seeds stuck in the sieve holes; 12 - second sieve angle adjustment system;

\section{3 - electric motor}

The first separation improvement is produced by the rotating sieve (1) in the opposite direction to the coil (2), so as to increase the level of agitation of the material and to be able to break the immobile core that is created in the operation of rotating sieves, usually decreasing the separation efficiency.

The inner coil is an element constructed of a customizable number of propellers fixed on a shaft (Fig 2 and 3). Each propeller can also be customized by adjusting two internal elements in order to obtain an even greater variation of the internal parameters. The screws (3) adjust the propeller blades (4), an element that determines the level of aggressiveness applied to the seeds. The screw (1) determines the attachment of the blade on the shaft, which regulates the pitch of the coil. 


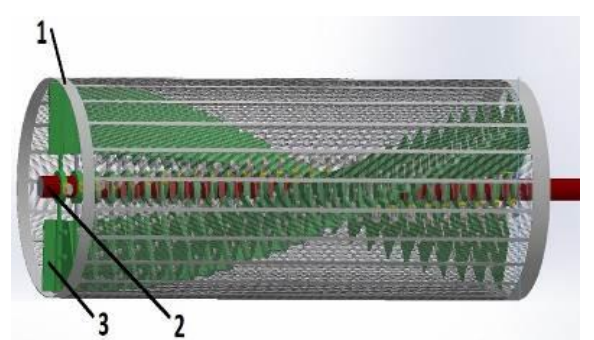

Fig. 2. Sieve and coil subassembly: $1-$ cylindrical sieve; 2 - shaft; 3 - inner coil

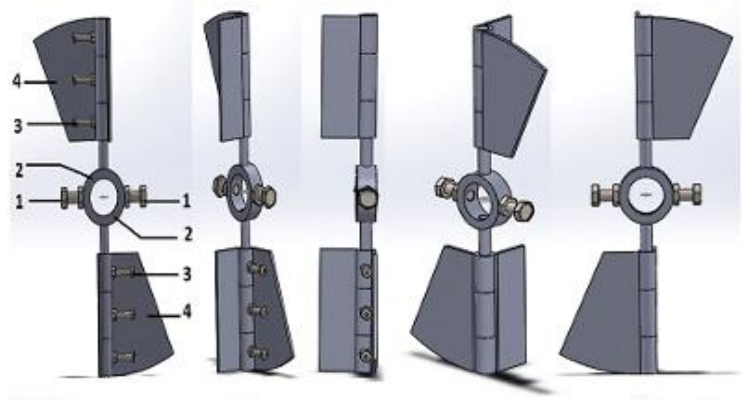

Fig. 3. Coil elements: 1 - shaft mounting screws; 2 - shaft socket; 3 - blade mounting screws; 4 - blades

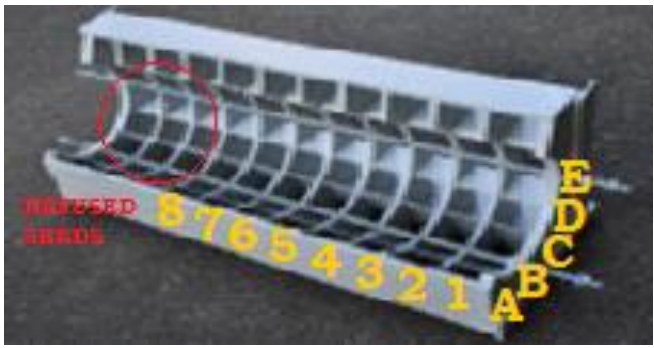

Fig. 4. Subassembly for collecting and quantifying the quantity of seeds separated in longitudinal plane (1-8) and angular plane (A-E), as well as large seeds (refused seeds)

The behavior of wheat seeds in rotating sieves, especially those equipped with inner coils, needs more in-depth studies to be able to determine how interior processes can be improved using new subassembly designs [7-9]. Inner coils can have many specific shapes and purposes and it is difficult and expensive to produce a wide range of models for testing and evaluation [10;11]. Therefore, one of our objectives was to produce for the pilot experimental stand a customizable inner coil model, which can be tested in different working regimes.

The angle between the two blades from each wing can be easily modified, since the large blades (1) and the small blades (2), are mounted in a hinge system and locked with fastening screws (5). By rotating the coil elements on the shaft the coil pitch can be established and varied, or one can opt for other forms of positioning, such as linear arrangement.

Changing the distance between the coil elements has an important role in the evaluation processes, because one can study the forces exerted inside the sieve when separating seeds with different characteristics.

\section{The methodology for conducting experiments}

The experiments described in the present paper aimed at separating non-conforming seeds that have dimensions below $2 \mathrm{~mm}$ from the mass of material. Seeds below $2 \mathrm{~mm}$ were considered impurities and have been colored with red paint for a better identification. In the first phase, a mixture of wheat seeds and impurities has been prepared, which totaled $1 \mathrm{~kg}$ (900 $\mathrm{g}$ of wheat and 100 grams of impurities).

The bunker shutter adjusts the flow of seeds entering the sieve to accurately determine the amount of seeds being processed. The shutter has been adjusted so that the amount of $1 \mathrm{~kg}$ of material to fall on the sieve within one minute and the rotational speed of the coil was set at 56 rotations per minute. The blades of the coil elements were set to an angle of 45 degrees, being adjusted at a distance of $1 \mathrm{~mm}$ from each other.

The mixture of wheat and impurities enters the sieve through the feeding door. Due to the rotational movement of the rotating sieve, the material advances by rolling and sliding. 
Impurities gradually pass through the sieve holes and accumulate in the collection boxes. The refused material represented by large seeds is collected separately. The varied parameter was the sieve inclination angle compared to the horizontal (the studied angles being: 1-degree; 3-degree and 5-degree).

The operation has been performed first without mounting the inner coil, after which the coil was associated to the subassembly and the measurements were repeated in order to be able to evaluate the difference in efficiency. Performing a comparative evaluation of the separating process, operating the equipment with and without the inner coil on a short sieve $(0.56 \mathrm{~m})$, we could evaluate some operating characteristics depending on the flow rate of seeds entering on the sieve, the angle of inclination of the sieve and the processing time of the material.

The studied sieve has the diameter of $200 \mathrm{~mm}$ and a length of $560 \mathrm{~mm}$. The distance between the moving blades and the sieve surface is $5 \mathrm{~mm}$, and the distance between the sieve and the collecting boxes is $3 \mathrm{~mm}$. The angle of blade displacement relative to each other was set to 45 degree. The coil elements were positioned at a distance of $1 \mathrm{~mm}$ from each other, in order not to allow the seeds to pass between the elements.

The experimental stage involved performing a number of 5 experiments for each case, in this study presenting the average of the obtained values.

\section{Results and discussion}

The first outcomes were oriented towards the design, production and validation of the performances of the pilot experiment stand, used to evaluate the processes exerted in the rotating cylindrical sieves. A challenging situation was assessed, when the sieve has small dimensions $(0.56 \mathrm{~m})$, and the separation must take place in a limited time, otherwise the material may remain unsorted.

The comparative evaluation of the process of separating contaminants in the pilot experimental stand, operating with an inner coil and without an inner coil, showed major differences depending on the parameters that were varied. Seeds separation along the sieve length (percentage distribution on the $0.56 \mathrm{~m}$ sieve) has been tested first for the inclination angle of 1degree deviated from the horizontal position and having a $10 \%$ contamination level (Figures 5 and 6). Changing the angle has the role of speeding up the sliding of the seeds and improving the processing time.

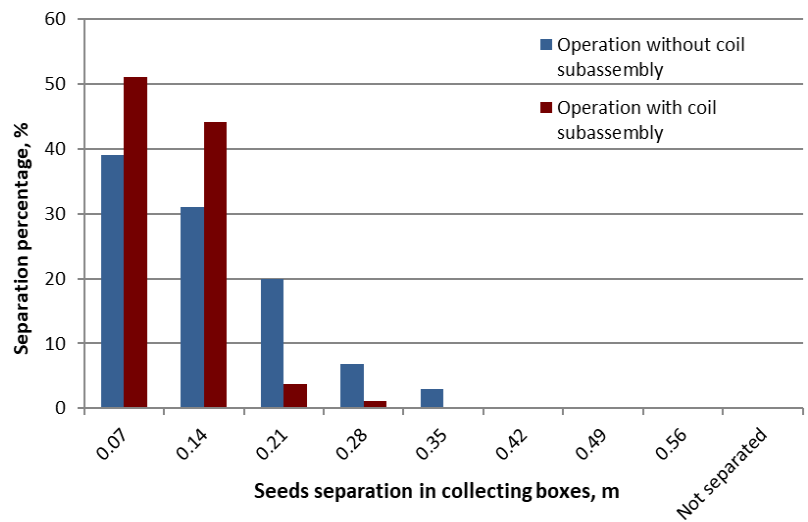

Fig. 5. Comparative evaluation of the equipment operating with an inner coil (red) and without an inner coil (blue), inclination of 1-degree from the horizontal

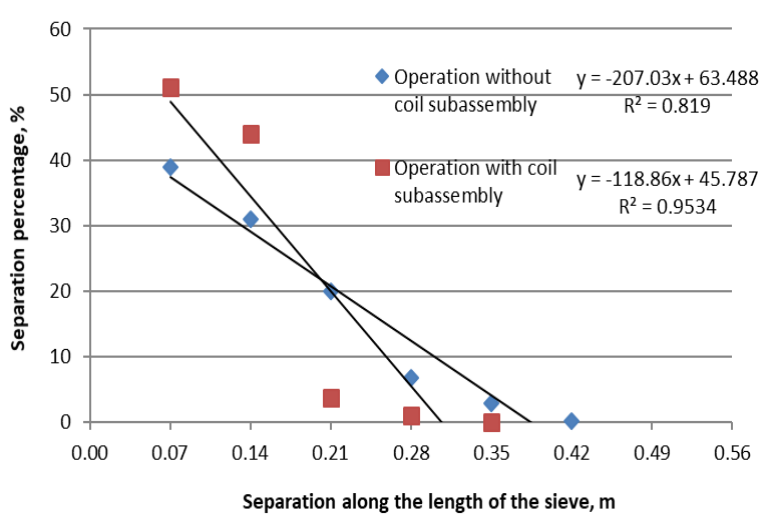

Fig. 6. Evaluation of separation along the side length with and without using the inner coil, for 1-degree inclination

Therefore, a quantity of $100 \mathrm{~g}$ of contaminant can be separated, using the pilot stand in the first two thirds of the sieve without the help of the coil. The angle of inclination of 1degree (compared to the horizontal) used in this case, make the movement of the seeds slow, so that the material subjected to separation will stay in the processing for a longer time. The bunker shutter has been adjusted so that the amount of $1 \mathrm{~kg}$ of material to fell on the sieve within one minute. The seeds spent a total of 5 minutes and 18 seconds on the sieve, until there was no material for processing.

The use of the coil (red color) makes the separation faster, the small seeds separating in the first third of the rotating sieve length. The sieve breaks the immobile core and has a tendency of pushing the seeds back, increasing the material separation time with 45 seconds. 
Although the separation was achieved completely, it can be seen that the process is not efficient. Therefore, either a higher flow rate must be set, or the speed of the seeds must be increased.

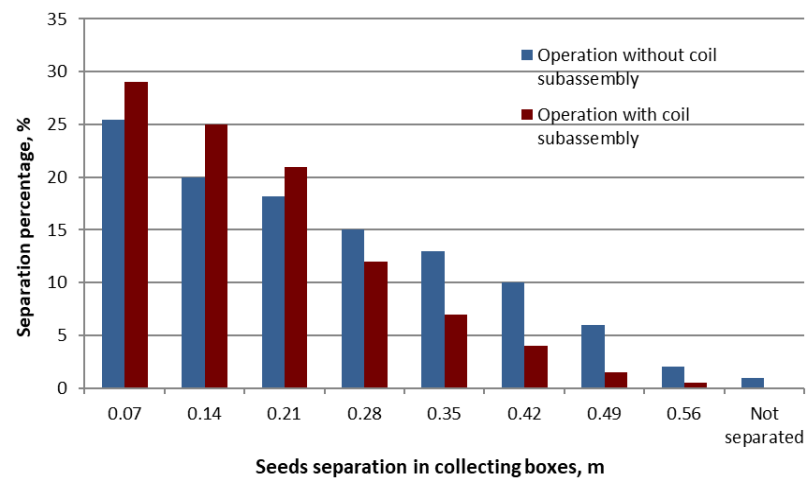

Fig. 7. Comparative evaluation of the equipment operating with an inner coil (red) and without an inner coil (blue), inclination of 3-degree from the horizontal

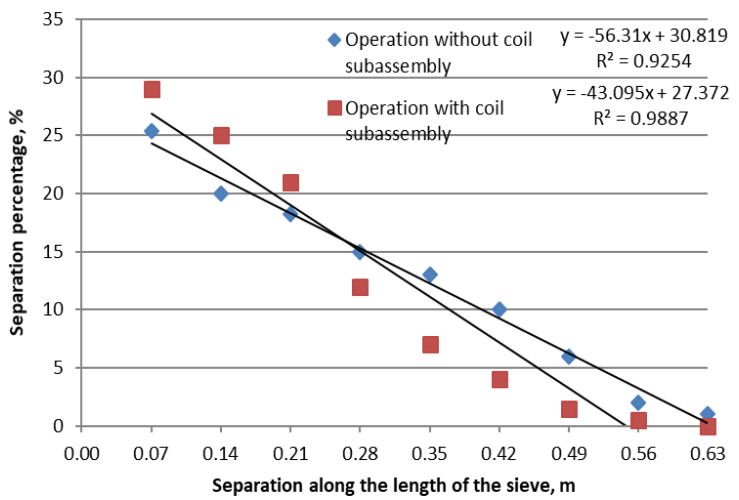

Fig. 8. Evaluation of separation along the side length with and without using the inner coil, for 3-degree inclination

For the case shown in Figures 7 and 8, the angle of inclination to the horizontal was increased to 3 degree, thus increasing the speed of the seeds found on the sieve surface. It can be seen that during the operation without having the internal coil mounted (blue color), the wheat does not separate completely. The reason is the small length of the sieve of only $0.56 \mathrm{~m}$, the seeds need more time to separate.

Operating the equipment, having the internal coil mounted (red color), the tendency of the coil to break the immobile core and to mix the seeds better makes the separation to be achieved completely. The separation time also decreased to 4 minutes and 15 seconds for coil-free operation and to 4 minutes and 25 seconds for operation with internal coil. The process is carried out in this case in conditions of increased efficiency.

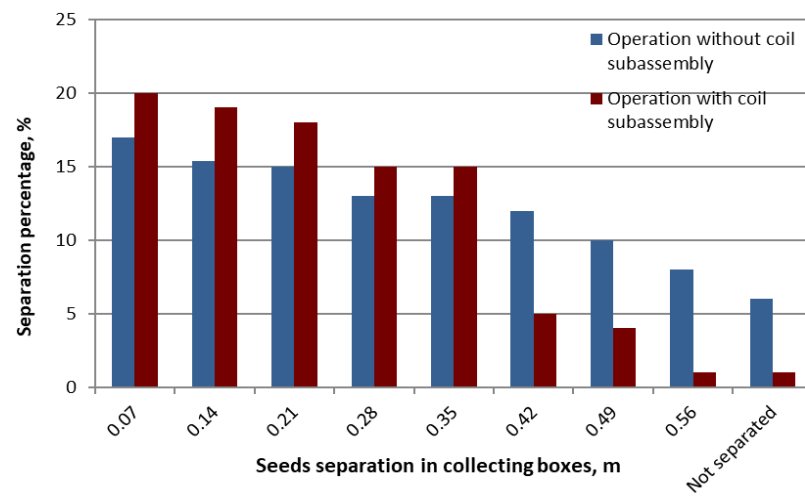

Fig. 9. Comparative evaluation of the equipment operating with an inner coil (red) and without an inner coil (blue), inclination of 5-degree from the horizontal

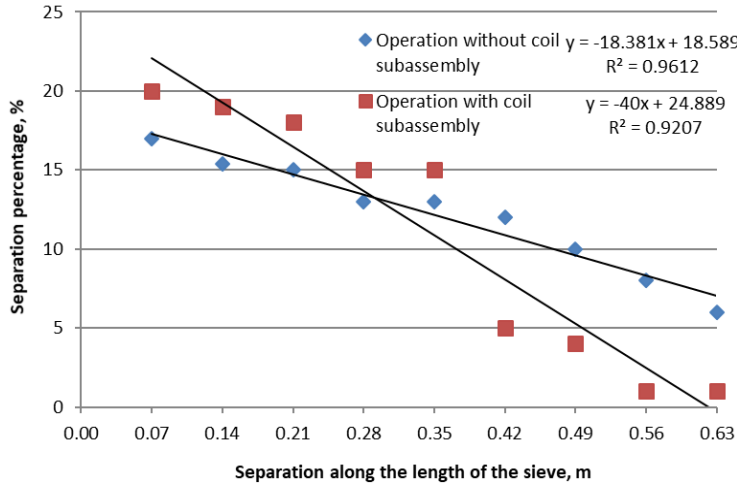

Fig. 10. Evaluation of separation along the side length with and without using the inner coil, for 5-degree inclination

Increasing even more the angle of inclination of the rotating sieve up to 5-degree compared to the horizontal, a more uniform distribution of the seed separation is obtained. When operating without a mounted coil an average quantity of $6 \%$ small seeds could not be separated in due time using the equipment. The use of the coil improved the process, making the amount of small seeds that were not separated to decrease to less than $1 \%$. The processing time has decreased for both cases under 4 minutes, and the efficiency of the sieve and coil subassembly is best highlighted by situations with high processing speeds and high flow rates of the processed material.

\section{Conclusions}

1. An experimental pilot stand was designed and constructed in order to determine the phenomena that occur during the separation of wheat seeds, depending on their size. Several subassemblies 
were tested, such as: an innovative sieve equipped with a customizable inner coil, a subassembly for testing the distribution of seeds collected along the sieve (collection boxes), and a system for driving the sieve and coil in opposite directions.

2. It has been determined that in the case of operating without the inner coil, the distribution of contaminating seeds on the surface of the sieve is constant. However, when the operation takes place at larger angles, the process takes place too fast to produce an efficient separation, and some contaminants fail to separate. The use of inner coil subassembly associated with the wheat separation equipment has shown very good results in the specific case of a short sieve. The highest improvement brought to the separation process by installing the inner coil has been identified for the 5-degree inclination angle. At this inclination, the seeds record the highest speed along the sieve length, therefore, the separation time is the shortest. In the case of using high inclination angles, the processing speeds have been counterbalanced by the use of the coil and driving subassemblies, so that the separation could be performed in better conditions.

\section{Acknowledgement}

This paper was supported by a grant offered by the Romanian Minister of Research as Intermediate Body for the Competitiveness Operational Program 2014-2020, call POC/78/1/2/, project number SMIS2014 + 136213, acronym METROFOOD-RO.

\section{References}

[1] Voicu G., Casandroiu T., Tarcolea C. Testing Stochastic Models for Simulating the Seeds Separation Process on the Sieves of a cleaning System, and a comparison with experimental data. Agriculturae Conspectus Scientificus, vol. 73, no. 2, 2008, pp. 95-101.

[2] Panturu D. Calculation and construction of equipment in the milling industry, Editura Tehnica, Bucuresti, 1997, 201 p.

[3] Mircea C., Nenciu F., Vlăduț V., Voicu G., Gageanu I., Cujbescu D. Increasing the performance of cylindrical separators for cereal cleaning, by using an inner helical coil, INMATEH - Agricultural Engineering, vol. 62, No. 3, 2020, pp. 249-258.

[4] Schreiber M., Kutzbach H.D. Modelling Separation Characteristics in Combine Cleaning Shoes, Landtechnik, no. 4, 2003, pp. 236-237;

[5] Banu C., Tratat de industrie alimentara. Tehnologii alimentare, Editura ASAB, 2009, 1152 p.

[6] Casandroiu T., Popescu M., Voicu G. A Developing a mathematical model for simulating the seeds separation process on the plane sieves, U.P.B. Sci. Bull., Series D, vol. 71, Iss. 3, 2009, pp. 17-28.

[7] Balascio C.C., Misra M.K., Johnson H.P. Particle Movement and Separation Phenomena for a Gravity Separator: I Development of a Markov Probability Model and Estimation of Model Parameters, American Society of Agricultural Engineers, Transactions of the ASAE, 1987, pp. 1834-1839.

[8] Yuan J., Wu C., Li H., Qi X., Xiao X., Shi X., Movement rules and screening characteristics of rice-threshed mixture separation through a cylinder sieve - Computers and Electronics in Agriculture 154, 2018, pp. 320-329.

[9] Naumenko M., Sokol S., Filipenko D., Guridova V., Kharytonov M.M., Numeric model of the grain mixture flow in a cylindrical sieve which revolves around the inclined axis - INMATEH-Agric. Eng., 2018 Vol.56 No.3, 2018, pp. 67-74.

[10] Shi X., Qi X., Wu C., Li H., Yuan J., Screening characteristics and parameter optimization of threshed rape mixture separation through a cylindrical sieve - International Agricultural Engineering Journal 27(3), 2018, pp. 53-64;

[11] Drumond A.A.L., Sales JD., Zuchi J., Camelo G.N., Souza M.M.V., Physiological quality of castor seeds (Ricinus communis L.) after processing - Journal of Seed Science, Vol. 41, Issue: 2, 2019, pp. 224-232. 